\title{
LA-UR-21-31286
}

Approved for public release; distribution is unlimited.

\section{Title: Partial program correctness}

Author(s): $\quad$ Herring, Stuart Davis

Intended for: WG21 teleconferences

Web

Issued: 
Disclaimer:

Los Alamos National Laboratory, an affirmative action/equal opportunity employer, is operated by Triad National Security, LLC for the National Nuclear Security Administration of U.S. Department of Energy under contract 89233218CNA000001. By approving this article, the publisher recognizes that the U.S. Government retains nonexclusive, royalty-free license to publish or reproduce the published form of this contribution, or to allow others to do so, for U.S. Government purposes. Los Alamos National Laboratory requests that the publisher identify this article as work performed under the auspices of the U.S. Department of Energy. Los Alamos National Laboratory strongly supports academic freedom and a researcher's right to publish; as an institution, however, the Laboratory does not endorse the viewpoint of a publication or guarantee its technical correctness. 


\title{
P1494R2: Partial program correctness
}

\author{
Audience: EWG; CWG; LEWG; WG14
}

S. Davis Herring <herring@lanl.gov>

Los Alamos National Laboratory

November 10, 2021

\section{History}

r2:

- Discussed the preexisting problem of input dependence

- Discussed interactions with other kinds of observable behavior

- Discussed lack of debugger support

- Fixed (preexisting) "first" in wording based on SG1 review

r1:

- Made introduction explicitly independent of contracts

- Fixed conditionals in example

\section{Problem}

Undefined behavior enables and extends many important optimizations (e.g., simplifying signed integer arithmetic and dead-code elimination). The "time travel" aspect of such optimizations (explicitly authorized by [intro.abstract]/5) is surprising to many programmers in that it can sometimes eliminate tests meant to detect the invalid operation in question. In particular, consider

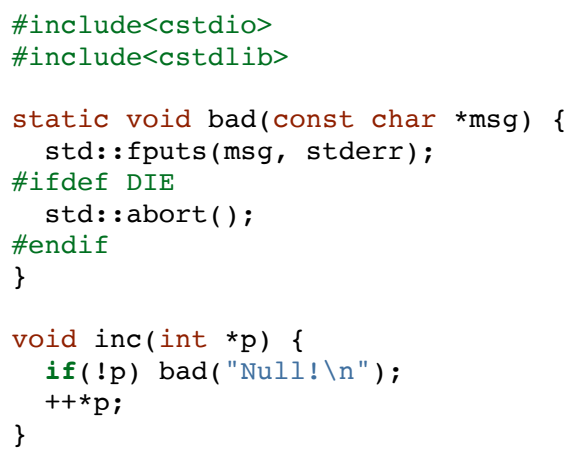

Without -DDIE, a conforming implementation can elide the test in inc entirely: std: : fputs always returns, so any call inc (nullptr) is guaranteed to have undefined behavior and need not call bad. (Note that current implementations do not do so in this case.)

This issue came up again recently in the discussion of contracts:

void $f($ int $* p)[[$ expects: p]] [ [expects: *p<5]];

Discomfort with the idea that (with continuation mode "on") the first contract-attribute-specifier might be elided because of the second was one of the motivations for the many late proposals to change (and eventually remove) contracts. Many wondered about the possibility of making a contract violation handler "opaque to optimization", so that the first precondition must be checked on the supposition that the handler might not return (but rather throw or terminate).

The capability of establishing such a "checkpoint", where subsequent program behavior, even if undefined, does not affect the preceding behavior, would be useful in general for purposes of stability and debugging.

There is already an analogous issue concerning program input: clearly a program can have undefined behavior for some inputs and not others. [intro.abstract] $/ 3$ and $/ 5$ acknowledge this by referring to "a given input" and "that input", but such a monolithic approach neglects the paradoxical possibility of input correlated with, say, unspecified quantities:

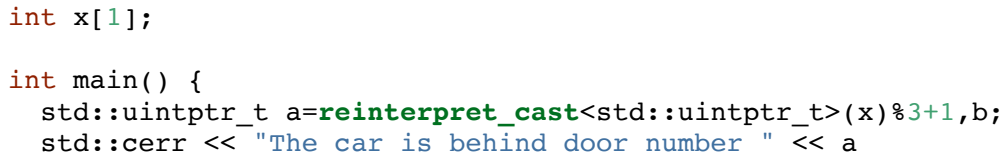


This program might suggest 2 (if the cast yields, say, 0x1000), but one could absurdly argue that responding with 2 leads to undefined behavior because almost all of the unspecified possibilities for the "address" of x (e.g., 0x2000) lead to undefined behavior for that input. An OOTA interpretation is also available: "a is really 3 , so the input 2 leads to undefined behavior, whose effect is to print 2 instead of a". We should reject this on the grounds of causality, since we require that undefined behavior respect input which can quite reasonably depend on prior output.

The standard does not have the necessary notion of prior, and this paper does not address this situation, but its checkpoints may be used to strengthen the guarantee of $/ 6.3$, that prompts are delivered before waiting for input, to include all observable behavior. This usage does exclude the OOTA interpretation: the output delivered to the host environment must accurately represent a if it is separated by a checkpoint from any potential undefined behavior.

\section{Previous work}

I suggested a trick involving a volatile variable, based on the idea that a volatile read is observable behavior ([intro.abstract]/6) that must be preserved by optimization.

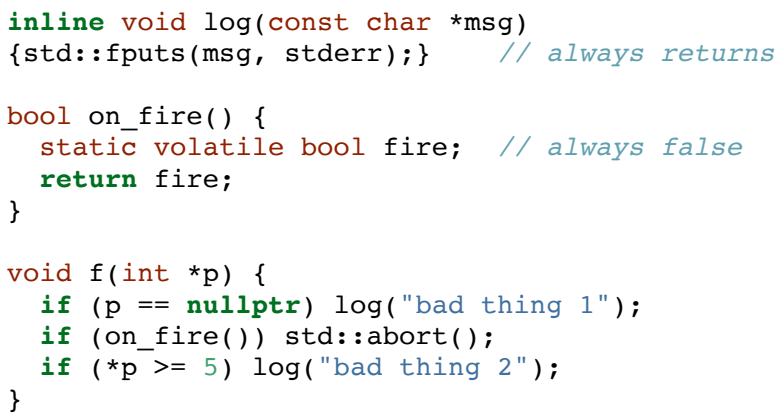

The idea is that the compiler cannot assume that on_fire ( ) returns false, and so the check for $p$ being null cannot be eliminated. However, the compiler can observe that, if $\mathrm{p}$ is null, the behavior will be undefined unless on fire () returns true, and so it can elide that check (though not the volatile read) and call abort ( ). This therefore seems to convey a certain capability of observing the upcoming undefined behavior without actually experiencing it.

Unfortunately, conforming implementations are not constrained to follow this analysis. It is logically necessary that the implementation perform the observable volatile read unless it can somehow obtain its result otherwise. However, after reading the value false (as of course it will be in practice) the implementation may take any action whatsoever, even "undoing" the call to log. For example, it would be permissible to perform the implicit flush for stderr only just before the call to std: : abort (which never happens). One might hope for the implementation to allow for the possibility that log affects some hardware state that affects the volatile read, but it might not as such a scheme would require support from the operating system.

\section{General solution}

We can instead introduce a special library function

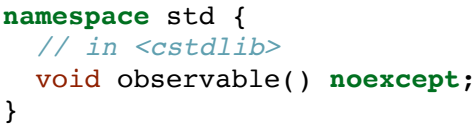

that divides the program's execution into epochs, each of which has its own observable behavior. If any epoch completes without undefined behavior occurring, the implementation is required to exhibit the epoch's observable behavior. Ending an epoch is nonetheless distinct from ending the program: for example, there is no automatic flushing of <cstdio $>$ streams.

Undefined behavior in one epoch may obscure the observable behavior of a previous epoch (for example, by re-opening an output file), but external mechanisms such as pipes to a logging process can be used to guarantee receipt of an epoch's output. With multiple threads, it is not the epochs themselves that are meaningful but their boundaries (or checkpoints); normal thread synchronization is required for the observable behavior of one thread to be included in an checkpoint defined by another.

As a practical matter, a compiler can implement std: : observable efficiently as an intrinsic that counts as a possible termination, which the optimizer thus cannot remove. After optimization (including any link-time optimization), the code generator can then produce zero machine instructions for it.

Note that std: : observable does not itself constitute observable behavior, and it does not forgive infinite empty loops ([intro.progress]/1). There 
Add paragraphs to the end of [support.start.term]:

- void observable() noexcept;

- Effects: Establishes an observable checkpoint ([intro.abstract]). No other effects. 